\title{
Can Campylobacter coli induce Guillain-Barré syndrome?
}

\author{
A. van Belkum • B. Jacobs • E. van Beek • R. Louwen • \\ W. van Rijs • L. Debruyne • M. Gilbert • J. Li • \\ A. Jansz • F. Mégraud • H. Endtz
}

Received: 4 October 2008 / Accepted: 17 October 2008/Published online: 12 November 2008

(C) The Author(s) 2008. This article is published with open access at Springerlink.com

Campylobacter jejuni enteritis is the most frequently identified infection preceding the Guillain-Barré syndrome (GBS) and neural damage is thought to be induced through molecular mimicry between $C$. jejuni lipo-oligosaccharide

A. van Belkum $(\triangle) \cdot$ E. van Beek $\cdot$ R. Louwen $\cdot$ H. Endtz

Department of Medical Microbiology and Infectious Diseases,

Erasmus MC,

's-Gravendijkwal 230,

3015 CE Rotterdam, The Netherlands

e-mail: a.vanbelkum@erasmusmc.nl

B. Jacobs $\cdot$ W. van Rijs

Departments of Neurology and Immunology, Erasmus MC,

's-Gravendijkwal 230,

3015 CE Rotterdam, The Netherlands

\section{Debruyne}

Laboratory for Microbiology, Ghent University,

K.L. Ledeganckstraat,

359000 Ghent, Belgium

M. Gilbert $\cdot$ J. Li

Institute for Biological Sciences,

National Research Council Canada,

100 Sussex Drive,

Ottawa, ON, Canada

A. Jansz

Laboratory for Public Health (PAMM),

Veldhoven, The Netherlands

F. Mégraud

Laboratoire de Bactériologie, Hôpital Pellegrin,

Place Amélie Raba-Léon,

33076 Bordeaux cedex, France

Present address:

H. Endtz

ICDDR,B: Laboratory Branch,

68 Shaheed Tajuddin Ahmed Sharani Mohakhali, Dhaka 1212,

GPO Box 128, Dhaka 1000, Bangladesh
(LOS) and human gangliosides [1]. It has been questioned whether or not other Campylobacter species, including $C$. curvus, C. upsaliensis and C. coli, could be similarly involved [2-4]. This is relevant because it would imply that bacterial factors considered important in the aetiology of GBS crossed species barriers. Two prior reports have appeared where $C$. coli was putatively associated with a case of GBS $[2,3]$.

We here present two female patients with GBS, one from the Netherlands (patient GB50) and one from France (patient 664H2004). From a faecal specimen obtained for both patients, a $C$. coli strain was isolated. On the basis of surface protein profiling, the strains were unequivocally demonstrated to belong to the species $C$. coli (results not shown). The strains were encoded GB50 and 664H2004, respectively, and stored at $-80^{\circ} \mathrm{C}$. For patient GB50, a serum sample obtained at the acute GBS phase was available. This sample was also stored at $-80^{\circ} \mathrm{C}$.

Strains were grown on Mueller-Hinton agar at $37^{\circ} \mathrm{C}$ for $48 \mathrm{~h}$, after which DNA was extracted, as described by Pitcher et al. [5]. Amplified fragment length polymorphism (AFLP) analysis was performed, as described by Duim et al. [6]. In brief, $1 \mu \mathrm{g}$ of genomic DNA was digested with the HindIII-HhaI restriction enzyme combination and sitespecific adaptors were ligated to the restriction fragments. Primers complementary to the adaptor and restriction site sequence were used in pre-selective and selective polymerase chain reaction (PCR) amplifications. The amplified and fluorescently labelled fragments were loaded on an ABI Prism 377 automated sequencer. GeneScan version 3.1 (Applied Biosystems) was used for data collection, and the AFLP profiles were imported, using the CrvConv filter, in BioNumerics 4.61 (Applied Maths, Belgium) for normalisation and further analysis. The obtained AFLP profiles were included in an in-house AFLP reference frame, containing 
profiles from all known Campylobacter species. Similarity between the normalised fingerprints was determined by the Pearson product moment correlation coefficient and a UPGMA dendrogram was constructed. The profiles from both isolates clearly formed a cluster together with $C$. coli reference strains (Fig. 1).

Using LOS gene cluster-specific PCR tests, the 664H2004 strain was demonstrated to harbour a B-type gene cluster [7]. For GB50, neither the $c s t 2$ nor the $c g t \mathrm{~A}$ gene was shown to be present. This convincingly demonstrates that this strain does not harbour the neuropathy-associated LOS gene cluster types A, B or C. Using primers based on the C. coli RM2228 LOS gene cluster, we amplified the LOS locus of GB50, but we could not amplify the LOS locus of $664 \mathrm{H} 2004$. Complete sequencing of the novel GB50 LOS gene cluster revealed 12 open reading frames (ORFs) that included five putative glycosyltransferases and a few ORFs seemingly unrelated to LOS biosynthesis (Table 1).

The LOS of C. coli strain GB50 did not bind cholera toxin, hence, the presence of a GM1-like ganglioside mimic could be excluded. The acute phase pre-treatment serum from patient GB50 showed a high level of IgG activity to the LOS from the GB50 strain (Table 2). This activity was significantly higher than in the serum from ten healthy blood donors. In addition, in the serum from patient GB50, IgM activity was found for this LOS, although it was less than the IgG activity. Some of the healthy blood donors also showed this elevated level of IgM. Probing the LOS with six specific monoclonal anti-ganglioside antibodies
(DG-1, DG-2, TBG-3, EG-7, EG-3 and EG-1) did not reveal any reactivity. These monoclonal antibodies were raised by immunisation with $C$. jejuni $\mathrm{LOS}$ and bound to various (combinations of) gangliosides (Table 2). Interestingly, regular mass spectrometry analysis on O-deacylated $C$. coli LOS [1] did not reveal a structural overlap between this LOS and the previously determined $C$. jejuni LOS structures. It appeared that the GB50 LOS did not contain sialic acid based on MS/MS analysis. The LOS from the 664H2004 strain was shown to contain di-NeuAc based on MS/MS analysis.

The serum from patient GB50 was tested in a standardised enzyme-linked immunosorbent assay (ELISA) for the detection of antibodies to the gangliosides GM1, GM2, GD1a, GD1b, GD3 and GQ1b [8]. Serum was positive for $\mathrm{IgG}$, IgM and IgA antibodies to the gangliosides GM1 and, to a lesser extent, to GD1b (Table 2). These gangliosides share the terminal $\mathrm{Gal}(\beta 1-3) \mathrm{GalNac}$ to which these antibodies are probably directed. Accordingly, the serum from this patient contained high IgG, IgM and IgA activity to the non-ganglioside glycolipid asialo-GM1, which has the same terminal disaccharide. Such a structure, however, was not identified in the LOS of the C. coli isolate from this patient. This suggests that this patient might have been coinfected with another organism expressing a GM1 mimic, possibly a $C$. jejuni strain, responsible for a non-GBSrelated induction of a cross-reactive antibody response to GM1. In inhibition ELISA experiments, anti-GM1 IgG activity in 1:400 dilutions of serum from GB50 was not reduced by LOS from this strain in concentrations of up to

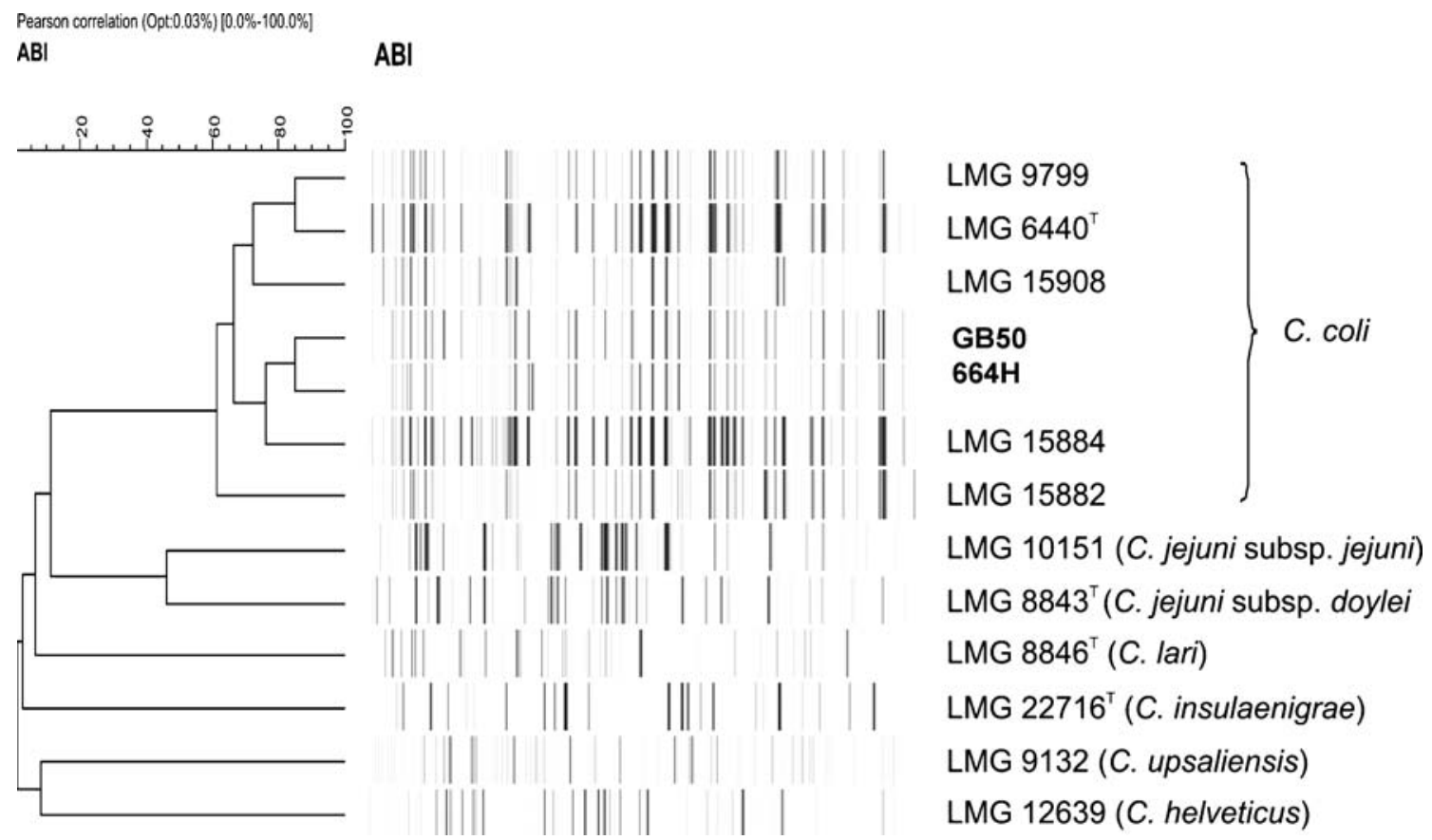

Fig. 1 Amplified fragment length polymorphism (AFLP) analysis of the two Campylobacter coli strains isolated from patients GB50 and 664H2004. Note that, despite their diverse geographic origins, these strains cluster, but clearly fall within the C. coli cluster 
Table 1 Information on the lipo-oligosaccharide (LOS) gene cluster for strain GB50 (GenBank accession number EU374214)

\begin{tabular}{lllll}
\hline Source sequence & & & $1 \ldots 11261$ & \\
\cline { 1 - 2 } Nucleotide position & Gene orientation & & Gene annotation & Putative gene function \\
\hline $1-108$ & + & waaC & Heptosyltransferase I \\
$101-988$ & + & htrB & Lipid A biosynthesis acyltransferase \\
$985-2538$ & + & orf3 & Putative glycosyltransferase \\
$2535-3587$ & + & orf4 & Putative glycosyltransferase \\
$3584-4783$ & - & orf5 & Putative glycosyltransferase \\
$4911-6215$ & + & orf6 & Putative CDP-glycerol:polyglycerophosphatase \\
$6212-7246$ & + & orf7 & Hypothetical protein \\
$7316-8335$ & + & orf8 & Transposase-like insertion element \\
$8214-8924$ & + & orf9 & Hypothetical protein \\
$8917-9837$ & + & orf10 & Putative DNA methyltransferase \\
$10021-10437$ & + & orf1 & Putative glycerol-3-phosphate cytidyltransferase. \\
$10456-11261$ & - & waaV & Putative glycosyltransferase \\
\hline
\end{tabular}

$200 \mu \mathrm{g} / \mathrm{ml}$, demonstrating that these antibodies do not crossreact with the LOS from the $C$. coli isolate from the patient. In control studies, this antibody activity was reduced to a level of less than $5 \%$ by pre-incubation with LOS from a $C$. jejuni isolate GB2 from another GBS patient, in which mimicry with GM1 was previously demonstrated by MS [9].

Overall, we have comprehensively characterised two $C$. coli strains isolated from GBS patients with a variety of state of the art technologies. One strain (664H2004) harboured disialylated LOS, but, unfortunately, no serum was available to measure either anti-ganglioside or antiLOS antibodies in the GBS patient herself. The other strain from the Dutch patient (GB50) had non-sialylated LOS, which showed no binding to cholera toxin or to monoclonals specific for several gangliosides. However, the serum from patient GB50 from which $C$. jejuni GB50 was isolated did contain antibodies to the LOS from this strain, indicating that this infection led to a specific immune response to C. coli strain GB50. The role of this immune response in the pathogenesis of GBS is currently unknown, although other examples of GBS patients infected with nonganglioside mimicking strains of $C$. jejuni have been described [10]. The serum from GB50, however, also contained antibodies to GM1 and GD1a, which do not

Table 2 Characterisation of Campylobacter coli strains isolated from two patients with Guillain-Barré syndrome (GBS)

\begin{tabular}{lll}
\hline & GB50 & $664 \mathrm{H} 2004$ \\
\hline $\begin{array}{l}\text { Genotyping strains } \\
\text { LOS biosynthesis cluster }\end{array}$ & Non-typable (no sialic acid incorporating enzymes encoded) & Class B (does contain Cst2) \\
Structure of LOS (MS/MS analysis) & - & Di-NeuAc \\
Presence sialic acids & - & - \\
Binding studies with LOS & - & - \\
Cholera toxin B-subunit & $++^{2}$ & - \\
Anti-ganglioside mAbs ${ }^{2}$ & $+^{2}$ & - \\
IgG in serum patient GB50 & GM1 (6400), GD1b (400) & \\
IgM in serum patient GB50 & GM1 (800), GD1b (200) & \\
Serum anti-ganglioside antibodies ${ }^{3}$ & GM1 (200), GD1b (100) & n.a. \\
IgG & & n.a. \\
IgM & - & - \\
IgA & & \\
Cross-reactivity serum antibodies & & \\
\hline Anti-GM1 IgG in serum GB50 & & \\
\hline
\end{tabular}

n.a.; not available

${ }^{1}$ Monoclonal antibodies DG-1 (to GM1), DG-2 (to GM1/GD1b/GA1), TBG-3 (to GD1a), EG-7 (to GD1b), EG-3 (to GQ1b) and EG-1 (to GT1a/ GQ1b)

${ }^{2}$ Significantly higher than antibody activity to this LOS in serum from ten healthy blood donors

${ }^{3}$ Serum IgG, IgM and IgA tested to GM1, GM2, GD1a, GD1b, GD3 and GQ1b

${ }^{4}$ The cross-reactivity of 1:400 diluted serum anti-GM1 IgG antibodies from patient GB50 to LOS from C. coli GB50 and $664 \mathrm{H} 2004$ were determined by inhibition enzyme-linked immunosorbent assay (ELISA) 
cross-react with LOS from GB50, as expected, although they do cross-react with LOS from a $C$. jejuni with a known GM1 mimic. These serological findings in patient GB50 strongly suggest that this patient was infected with at least two micro-organisms, one of which induced the antiganglioside antibodies leading to GBS (possibly a C. jejuni strain), and $C$. coli, which also induced an immune response, but probably did not trigger GBS.

In summary, we could not produce any evidence that molecular mimicry plays a role in the pathogenesis of GBS that is preceded by a $C$. coli infection. It is still not clear whether C. coli isolated from the faeces of GBS patients can play a role in the development of GBS. Additional GBS patients and their $C$. coli strains need to be reported and characterised in detail in order to better understand the pathogenesis of GBS in these patients.

Acknowledgements Eeti van Beek was supported by a grant from the Dutch Digestive Foundation (MWO 05-27). Jean Louis Gaillard (Hopital Poincaré, Garches, France) provided the clinical isolate of Campylobacter coli 664H2004. We thank Marie France Karwaski and Denis Brochu (both from the National Research Council Canada) for their technical help with the LOS NMR analysis. Prof. Hugh Willison (Division of Clinical Neurosciences, Glasgow Biomedical Research Centre, University of Glasgow, Glasgow, UK) is acknowledged for providing the monoclonal antibodies used in the present study.

Open Access This article is distributed under the terms of the Creative Commons Attribution Noncommercial License which permits any noncommercial use, distribution, and reproduction in any medium, provided the original author(s) and source are credited.

\section{References}

1. Dzieciatkowska M, Brochu D, van Belkum A, Heikema AP, Yuki N, Houliston RS, Richards JC, Gilbert M, Li J (2007) Mass spectrometric analysis of intact lipooligosaccharide: direct evidence for O-acetylated sialic acids and discovery of O-linked glycine expressed by Campylobacter jejuni. Biochemistry 46:14704-14714. doi:10.1021/bi701229k

2. Bersudsky M, Rosenberg P, Rudensky B, Wirguin I (2000) Lipopolysaccharides of a Campylobacter coli isolate from a patient with Guillain-Barré syndrome display ganglioside mimicry. Neuromuscul Disord 10:182-186. doi:10.1016/S0960-8966(99) 00106-6

3. Funakoshi K, Koga M, Takahashi M, Hirata K, Yuki N (2006) Campylobacter coli enteritis and Guillain-Barré syndrome: no evidence of molecular mimicry and serological relationship. J Neurol Sci 246:163-168

4. Koga M, Yuki N, Takahashi M, Saito K, Hirata K (1999) Are Campylobacter curvus and Campylobacter upsaliensis antecedent infectious agents in Guillain-Barré and Fisher's syndromes? J Neurol Sci 163:53-57. doi:10.1016/S0022-510X(98)00332-3

5. Pitcher DG, Saunders NA, Owen RJ (1989) Rapid extraction of bacterial genomic DNA with guanidium thiocyanate. Lett Appl Microbiol 8:151-156. doi:10.1111/j.1472-765X.1989.tb00262.x

6. Duim B, Wassenaar TM, Rigter A, Wagenaar J (1999) Highresolution genotyping of Campylobacter strains isolated from poultry and humans with amplified fragment length polymorphism fingerprinting. Appl Environ Microbiol 65:2369-2375

7. Taboada EN, van Belkum A, Yuki N, Acedillo RR, Godschalk PC, Koga M, Endtz HP, Gilbert M, Nash JH (2007) Comparative genomic analysis of Campylobacter jejuni associated with Guillain-Barré and Miller Fisher syndromes: neuropathogenic and enteritis-associated isolates can share high levels of genomic similarity. BMC Genomics 8:359. doi:10.1186/14712164-8-359

8. Kuijf ML, Van Doorn PA, Tio-Gillen AP, Geleijns K, Ang CW, Hooijkaas H, Hop WC, Jacobs BC (2005) Diagnostic value of anti GM1 ganglioside serology and validation of the INCAT-ELISA. J Neurol Sci 239:37-44. doi:10.1016/j.jns.2005.07.009

9. Godschalk PC, Heikema AP, Gilbert M, Komagamine T, Ang CW, Glerum J, Brochu D, Li J, Yuki N, Jacobs BC, van Belkum A, Endtz HP (2004) The crucial role of Campylobacter jejuni genes in anti-ganglioside antibody induction in Guillain-Barré syndrome. J Clin Invest 114:1659-1665

10. Godschalk PC, Kuijf ML, Li J, St Michael F, Ang CW, Jacobs BC, Karwaski MF, Brochu D, Moterassed A, Endtz HP, van Belkum A, Gilbert M (2007) Structural characterization of Campylobacter jejuni lipooligosaccharide outer cores associated with Guillain-Barré and Miller Fisher syndromes. Infect Immun 75:1245-1254. doi:10.1128/IAI.00872-06 